\title{
Redes Semánticas de la Pobreza. La Argentina del 2001
}

\author{
Gerardo Avalle - Universidad Católica de Córdoba - Facultad de Ciencia política y \\ Relaciones Internacionales ${ }^{1}$
}

\section{Resumen}

El presente trabajo se enmarca dentro de la corriente cualitativa del análisis de redes, donde se intenta reconstruir la estructura global de las redes semánticas sobre determinados fenómenos de la realidad social. Se pretende trabajar con las noticias aparecidas en el diario La Voz del Interior en el año 2001 referidas a la pobreza, este periódico se edita en la ciudad de Córdoba, República Argentina. El objetivo que transita sobre este texto es identificar las redes "semánticas" de la pobreza, en un período significativo, de una base original de 1600 artículos correspondientes al período 1990-2001 son trabajados aquellos correpondientes al último año. Este año se presenta como una quiebra en el "ciclo de protestas" sociales (Tarrow, 1997) en Argentina, marcando un hito en el proceso de las luchas populares en Argentina.

Palabras clave: pobreza, red semántica, discurso, Estado.

\begin{abstract}
This paper is framed within the qualitative stream of network analysis, trying to rebuild the global structure of the semantics of networks events. The data is based in the news appeared in the newspaper "La Voz del Interior" during the year 2001 about poverty, being this newspaper published in the city of Cordoba, Republic of Argentina. The objective on this text is to identify "the semantic" networks of the poverty in a significant period selecting from the 1600 articles corresponding to period of 1990-2001 those correspondent to the last year. This year is a break in the "cycle of social protests" (Tarrow, 1997) in Argentina, a landmark in the process of the popular struggles in Argentina.
\end{abstract}

Key words: poverty, semantic network, discourse, State.

El presente trabajo intenta dar cuenta de la potencialidad que posee el análisis de redes como herramienta metodológica para analizar los discursos sociales. Tal es así que a partir de la lectura de notas del diario de mayor tirada en la provincia se han construido numerosas redes que dan cuenta de los problemas que emergen en torno al concepto de pobreza, los actores y la configuración de posibles escenarios de conflictos.

\footnotetext{
1 Enviar correspondencia a: avallegera@hotmail.com. Becario Conicet-Agencia Córdoba Ciencia, Licenciado en Ciencia Política (UCC). Mestrando en Sociología (UNC) J efe de Trabajos Prácticos de la cátedra de Metodología I, de la Facultad de Ciencia Política y Relaciones Internacionales (UCC). Investigador (UCC) del proyecto "El llano en llamas: luchas sociales urbanas y campesina en la Córdoba de hoy" a cargo de la Mgter. Alejandra Ciuffolini.
} 
Se trabajaron 136 notas del año 2001 referentes a la pobreza, identificando como unidad de análisis los tópicos a la que cada una de ellas refiere, donde la construcción de las categorías de análisis fue posterior a la lectura de las mismas. A su vez se han identificado lo actores generadores de las mismas, con lo que se pretende trabajar las relaciones que aparezcan entre estos dos elementos, la importancia de los mismos y la identificación de los diferentes nodos semánticos. Propósitos que pueden ser abordados mediante las herramientas que proporciona el análisis de redes.

Se supone una noción reticular entre los distintos elementos textuales que puedan aparecer, esto es, no se trabaja con los atributos del texto, sino con los significados que puedan ser otorgados a él. Existe una idea central que atraviesa todo el análisis, y es la importancia del discurso como constructor de realidades. El término "discurso" no se circunscribe al lenguaje oral o escrito, sino que se refiere a la relación actual entre un conjunto de signos (de distinta naturaleza) pertenecientes a un determinado universo que a su vez se vincula (concordando u oponiéndose) con otros universos (formaciones discursivas).

La categorías con las que se cuentan permiten obtener la superestructura textual de cada nota y agruparlas según las coincidencias tópicas, pero o se ha analizado aún su conexión global en la red de la pobreza, ni las implicancias que puedan tener determinadas vinculaciones entre los actores. Este posicionamiento relacional y no atributivo supone la irreductibilidad del análisis social más allá de la interacción, de la relación, considerando por ello que todo intento de homologar las características individuales de actores, tópicos, etc, a un corpus global genera serias dificultades en su reproducción de lo real. Se puede afirmar entonces la intención de construir una red como señala Lozares Colina donde "la personas constituyen los nodos (...) y los datos verbales o textuales son tomados como relación que une a esos actores" (2002:5).

El vínculo presente entre los diferentes tópicos o nodos va a estar manifestando la presencia de microestructuras temáticas y los posicionamientos discursivos de los actores. Un primer análisis y de modo totalmente descriptivo es otorgado por el programa SPSS donde se enumeran tanto actores como temas de las notas analizadas. 


\begin{tabular}{|c|c|c|}
\hline Actores & Frecuencia & Porcentaje Válido \\
\hline SOCIEDAD CIVIL NO ORGANIZADA & 7 & 5,1 \\
\hline I GLESI A ( PUEBLO, OTRAS ORGANI ZACI ONES) & 15 & 11,0 \\
\hline DESEMPLEADOS & 3 & 2,2 \\
\hline ORG / CONFERENCI AS I NTERNACI ONALES & 10 & 7,4 \\
\hline GOB. PCIA DE CBA. & 8 & 5,9 \\
\hline GOB. NACIONAL & 11 & 8,1 \\
\hline GoB. MUNICIPAL (CBA. Y OTROS) & 7 & 5,1 \\
\hline UNIVERSIDADES Y SUS DEPENDENCIAS & 1 &, 7 \\
\hline SINDICATOS Y SUS CONFEDERACIONES & 6 & 4,4 \\
\hline ORG. VARI AS DE LA SOC. CIVI L & 21 & 15,4 \\
\hline SECTOR PRIVADo (EMPRESARIO) & 3 & 2,2 \\
\hline ESTUDI ANTES & 1 &, 7 \\
\hline ESCUELAS EN GRAL. Y DOCENTES & 2 & 1,5 \\
\hline ALFONSÍN & 1 &, 7 \\
\hline VOLUNTARIOS & 6 & 4,4 \\
\hline Sectores Carenci ados, Pobres & 10 & 7,4 \\
\hline NIÑOS, ADOLESCENTES & 2 & 1,5 \\
\hline 56 & 4 & 2,9 \\
\hline PROFESIONALES & 7 & 5,1 \\
\hline OPINIÓN PÚBLICA & 1 &, 7 \\
\hline USTICIA & 1 &, 7 \\
\hline PIQUeTERos & 9 & 6,6 \\
\hline Total & 136 & 100 \\
\hline
\end{tabular}

Tabla 1. Actores más relevantes.

\begin{tabular}{|c|c|c|}
\hline Tema Principal & Frecuencia & Porcentaje Válido \\
\hline DEMANDA DE RECURSOS FINANCIEROS & 3 & 2,2 \\
\hline DEMANDA DE BIENES Y SERVICIOS & 7 & 5.1 \\
\hline DEMANDA de PACto ( GENÉri CO O AMBos) & 7 & 5,1 \\
\hline CRÍ STICAS O DENUNCIAS SOBRE LA SITUACIÓN & 57 & 41.9 \\
\hline CRIticas o denuncias a políticas & 19 & 14,0 \\
\hline CRITICAS O DENUNCIAS IDEOLÓGICAS & 3 & 2,2 \\
\hline DENUNCIAS DIRIGIDAS AL ESTADO & 2 & 1,5 \\
\hline OFERTA DE PP PLANES SOCIALES & 5 & 3,7 \\
\hline OFERTA DE PP PROYECTOS ESPECÍFICOS & 4 & 2,9 \\
\hline OFERTA DE PP SERVICIOS TRADICIONALES & 2 & 1,5 \\
\hline OFERTA DE PP SERVICIOS NUEVOS & 1 &, 7 \\
\hline I NI CI ATI VAS Y POLÍ TI CAS SURGI DAS EN LA SOC. & 12 & 8,8 \\
\hline I NICIATIVAS Y POLÍTICAS SURGIDAS EN LA SOC. PROMO & 3 & 2,2 \\
\hline I NICIATIVAS Y POLÍTICAS SURGIDAS EN OI ASISTENCIA & 1 & , 7 \\
\hline I NICIATIVAS Y POLÍTICAS SURGIDAS EN OI PROMOCION & 1 & , 7 \\
\hline RECONOCIMIENTOS, APOYOS A PROGRAMAS & 4 & 2,9 \\
\hline DENUNCIAS DIRIGIDAS A POLÍTICOS A NIVEL MUNICIPAL & 1 & , 7 \\
\hline DENUNCIAS DIRIGIDAS A DOCENTES & 1 &, 7 \\
\hline DENUNCIAS DIRIGIDAS A MOVIMIENTOS POPULARES & 2 & 1,5 \\
\hline RECONOCIMIENTOS, APOYOS A POLÍTICAS COMUNITARIAS & 1 & 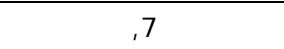 \\
\hline Total & 136 & 100 \\
\hline
\end{tabular}

Tabla 2. Temas más relevantes. 


\begin{tabular}{|c|c|c|c|c|c|c|c|c|}
\hline & $\begin{array}{c}\text { Demanda } \\
\text { s de Rec, } \\
\text { bs y pacto }\end{array}$ & Críticas & $\begin{array}{l}\text { Denuncias } \\
\text { al Estado }\end{array}$ & $\begin{array}{c}\text { Denuncias a } \\
\text { Movi- } \\
\text { mientos } \\
\text { Pop }\end{array}$ & $\begin{array}{l}\text { Oferta de } \\
\text { Políticas } \\
\text { Públicas }\end{array}$ & $\begin{array}{c}\text { I niciativas } \\
\text { y Pol. de la } \\
\text { Soc. Civil }\end{array}$ & $\begin{array}{l}\text { I nic. y Pol. } \\
\text { de Org. int. }\end{array}$ & $\begin{array}{c}\text { Reconocimi } \\
\text { entos y } \\
\text { apoyos a } \\
\text { Políticas }\end{array}$ \\
\hline $\begin{array}{l}\text { Soc. Civil no } \\
\text { Organizada }\end{array}$ & 5 & 0 & 0 & 2 & 7 & 0 & 1 & 1 \\
\hline I glesia & 0 & 0 & 0 & 0 & 0 & 0 & 2 & 0 \\
\hline Desempleados & 0 & 0 & 0 & 0 & 1 & 0 & 0 & 0 \\
\hline Gob. pcia de Cba. & 2 & 0 & 0 & 0 & 0 & 0 & 0 & 0 \\
\hline Gob. Nacional & 0 & 0 & 0 & 0 & 0 & 0 & 0 & 0 \\
\hline Gob. Municipal & 1 & 0 & 0 & 0 & 5 & 0 & 0 & 0 \\
\hline Universidades & 9 & 0 & 0 & 0 & 0 & 0 & 0 & 0 \\
\hline Sindicato & 1 & 0 & 0 & 0 & 1 & 0 & 0 & 0 \\
\hline $\begin{array}{l}\text { Org. de la Soc. } \\
\text { Civil }\end{array}$ & 7 & 0 & 0 & 0 & 0 & 0 & 0 & 1 \\
\hline Sector Privado & 1 & 0 & 0 & 0 & 0 & 0 & 0 & 2 \\
\hline Estudiantes & 1 & 0 & 0 & 0 & 0 & 0 & 0 & 0 \\
\hline Docentes & 8 & 0 & 0 & 0 & 0 & 0 & 0 & 0 \\
\hline Alfonsin & 8 & 0 & 0 & 0 & 0 & 1 & 1 & 0 \\
\hline Voluntarios & 0 & 1 & 0 & 0 & 0 & 5 & 0 & 0 \\
\hline $\begin{array}{l}\text { Sectores } \\
\text { Carenciados }\end{array}$ & 1 & 9 & 0 & 0 & 0 & 0 & 0 & 0 \\
\hline $\begin{array}{l}\text { Niños, } \\
\text { Adolescentes }\end{array}$ & 0 & 1 & 0 & 0 & 0 & 1 & 0 & 0 \\
\hline Profesionales & 0 & 7 & 0 & 0 & 0 & 0 & 0 & 0 \\
\hline Opinión Pública & 0 & 1 & 0 & 0 & 0 & 0 & 0 & 0 \\
\hline J usticia & 0 & 1 & 0 & 0 & 0 & 0 & 0 & 0 \\
\hline Piqueteros & 1 & 8 & 0 & 0 & 0 & 0 & 0 & 0 \\
\hline Org. I nternac. & 0 & 8 & 0 & 0 & 0 & 0 & 1 & 1 \\
\hline
\end{tabular}

Tabla 3. Matriz de actores por temas.

En el texto se encuentran remarcados aquellos tópicos o actores que registran mayor frecuencia de aparición. Un análisis de coocurrencia será efectuado a través del programa UCINET.

Se procedió con la construcción de una matriz "J" mediante los datos otorgados por el procedimiento de cross tabulation de estas dos dimensiones de los textos, "actores" y "eventos" (temas). 


\begin{tabular}{|c|c|c|c|c|c|c|c|c|c|c|c|c|c|c|c|c|c|c|c|c|c|}
\hline & $\begin{array}{r}1 \\
S \infty\end{array}$ & $\operatorname{Igl}$ & Des & Gob & $\begin{array}{r}5 \\
\mathrm{Gob}\end{array}$ & $\begin{array}{r}6 \\
\text { Gob }\end{array}$ & Uni & $\operatorname{Sin}^{8}$ & $\begin{array}{r}9 \\
\text { Org }\end{array}$ & 10 & $\begin{array}{r}11 \\
\text { Est }\end{array}$ & $\begin{array}{r}12 \\
D O C\end{array}$ & $\begin{array}{r}13 \\
\text { Alf }\end{array}$ & $\begin{array}{r}14 \\
\text { Vol }\end{array}$ & $\begin{array}{l}15 \\
\sec \end{array}$ & $\begin{array}{r}16 \\
\text { Niß }\end{array}$ & $\begin{array}{r}17 \\
\text { Pro }\end{array}$ & $\begin{array}{r}18 \\
\text { Opi }\end{array}$ & $\begin{array}{r}19 \\
\text { Jus }\end{array}$ & $\begin{array}{r}20 \\
\text { Piq }\end{array}$ & $\begin{array}{r}21 \\
\text { Org }\end{array}$ \\
\hline 1 Soc. Civil no Organizada & 21 & 34 & 12 & 10 & 24 & 12 & 4 & 20 & 21 & 2 & 0 & 8 & 0 & 4 & 36 & 4 & 28 & 4 & 4 & 32 & 3 \\
\hline Iglesia & 34 & 77 & 24 & 20 & 52 & 27 & 8 & 43 & 65 & 3 & 1 & 16 & 3 & 13 & & 9 & & & 8 & 67 & \\
\hline Gob Desenpleados & 12 & 24 & $\begin{array}{l}9 \\
6\end{array}$ & ${ }_{23}^{6}$ & 18 & 9 & 3 & 15 & 15 & 0 & $\begin{array}{l}0 \\
0\end{array}$ & 6 & 0 & 3 & 19 & 3 & 21 & 3 & 3 & 24 & $\frac{4}{6}$ \\
\hline $\begin{array}{l}\text { Gob. pela de Cba } \\
\text { Gob. Nacional }\end{array}$ & 24 & $\begin{array}{l}20 \\
52\end{array}$ & $\begin{array}{r}6 \\
18\end{array}$ & 22 & $\begin{array}{l}25 \\
47\end{array}$ & $\begin{array}{l}19 \\
28\end{array}$ & $\frac{2}{6}$ & $\begin{array}{l}11 \\
31\end{array}$ & $\begin{array}{l}24 \\
42\end{array}$ & $\begin{array}{l}1 \\
1\end{array}$ & 0 & 12 & $\begin{array}{l}1 \\
1\end{array}$ & $\frac{2}{6}$ & $\begin{array}{l}19 \\
55\end{array}$ & $\frac{2}{6}$ & $\begin{array}{l}14 \\
42\end{array}$ & $\frac{2}{6}$ & $\frac{2}{6}$ & $\begin{array}{l}17 \\
49\end{array}$ & 18 \\
\hline Gob. Municipal & 12 & 27 & 9 & 19 & 28 & 19 & 3 & 16 & 27 & 7 & 0 & 6 & 1 & 3 & 28 & 3 & 21 & 3 & 3 & 25 & 4 \\
\hline Universidades & 4 & 8 & 3 & 2 & 6 & 3 & 1 & 5 & 5 & 0 & 0 & 2 & 0 & 1 & 9 & 1 & 7 & 1 & 1 & 8 & \\
\hline Sindicatos & 20 & 43 & 15 & 11 & 31 & 16 & 5 & 26 & 31 & & 0 & 10 & 1 & 5 & 46 & 5 & 35 & & 5 & 41 & 0 \\
\hline Org, de la Soc. Civil & 21 & 65 & 15 & 24 & 42 & 27 & 5 & 31 & 115 & 8 & 7 & 10 & 6 & 40 & 51 & 12 & 35 & & 5 & 46 & 1 \\
\hline Sector Privado & 2 & 3 & 0 & 1 & 1 & 1 & 0 & 1 & 8 & & 0 & 0 & 1 & 0 & 1 & 0 & 0 & & & 1 & \\
\hline Estudiantes & $\mathbf{0}$ & 1 & 0 & 0 & 0 & 0 & 0 & 0 & 7 & 0 & 1 & 0 & 0 & 5 & 0 & 1 & 0 & 0 & 0 & 0 & 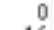 \\
\hline Docentes & 8 & 16 & 6 & 4 & 12 & 6 & 2 & 10 & 10 & & $\mathbf{0}$ & 4 & 0 & 6 & 18 & & 14 & & & 16 & 6 \\
\hline Alfonsin & 0 & 3 & 0 & 1 & 1 & 1 & $\overline{0}$ & 1 & 6 & & 0 & & 1 & 0 & 1 & $\overline{0}$ & 0 & & & 1 & \\
\hline Voluntarios & 4 & 13 & 3 & 2 & 6 & 3 & 1 & 5 & 40 & & 5 & 2 & 0 & 26 & 9 & 6 & 7 & 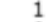 & 1 & 8 & 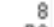 \\
\hline Sectores Care: & 36 & 75 & 27 & 19 & 55 & 28 & 9 & 46 & 51 & & $\mathbf{0}$ & 18 & & 9 & 82 & & 63 & & & 73 & 72 \\
\hline Niños, Adolescentes & 4 & 9 & 3 & 2 & 6 & 3 & 1 & 5 & 12 & & 1 & 2 & 0 & 6 & 9 & 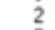 & 7 & 1 & 1 & 8 & 6 \\
\hline Profesionales & 28 & 56 & 21 & 14 & 42 & 21 & 7 & 35 & 35 & 0 & 0 & 14 & $\mathbf{0}$ & 7 & 63 & 7 & 49 & 7 & & 56 & 56 \\
\hline Opinión Pública & 4 & & 3 & 2 & 6 & 3 & 1 & 5 & 5 & & 0 & 2 & & 1 & 9 & & 7 & & & 8 & \\
\hline Justicia & 4 & 8 & 3 & 2 & 6 & 3 & $i$ & 5 & 3 & & 0 & & 0 & 1 & 9 & $i$ & 7 & 1 & 1 & 8 & 8 \\
\hline Piqueteros & 32 & 67 & 24 & 17 & 49 & 25 & $\therefore$ & 41 & 46 & & 0 & 16 & & 8 & 73 & & 56 & & & 65 & 4 \\
\hline Org. Internac. & 33 & 65 & 24 & 16 & 48 & 24 & 8 & 40 & 41 & 2 & 0 & 16 & 0 & 8 & 72 & 8 & 56 & 8 & 8 & 64 & 6 \\
\hline
\end{tabular}

Tabla 4. Matriz de Co-ocurrencia de actores.

Esta tabla nos permite observar la coincidencia de los diferentes actores en determinados lexemas o significantes de la pobreza. Del mismo podemos destacar las más importantes que son: I glesia, con el Gobierno nacional, las Organizaciones de la Sociedad Civil, los Sectores Carenciados, con los Grupos Piqueteros y las Organizaciones Internacionales. La Sociedad Civil no Organizada con la Iglesia, los Sectores Carenciados, Los Grupos Piqueteros y las Organizaciones Internacionales. Los Desempleados con la Iglesia, los Sectores Carenciados, Ios Profesionales, con los Grupos Piqueteros y las Organizaciones Internacionales. El Gobierno Provincial con la Iglesia, el Gobierno Nacional y con Organizaciones de la Sociedad Civil. El Gobierno Nacional con la Iglesia, los Sindicatos, los Sectores Carenciados y los Grupos Piqueteros. Las Organizaciones de la Sociedad Civil con la Iglesia, el Gobierno Nacional, los Sectores Carenciados y con los Grupos Piqueteros. Los Sectores Carenciados con la Iglesia, el Gobierno Nacional, las Organizaciones de la Sociedad Civil, los Profesionales, los Grupos Piqueteros y con las Organizaciones Internacionales. Por último, los Grupos Piqueteros con el Gobierno Nacional, la Iglesia, las Organizaciones de la Sociedad Civil, los Sectores Carenciados y con las Organizaciones Internacionales. 


\begin{tabular}{|c|c|c|c|c|}
\hline & & Degree & NrmDegree 2 & Share \\
\hline 15 & Sectores Carenciados & 610.000 & 3050.000 & 0.107 \\
\hline 2 & $\begin{array}{r}\text { Iglesia }\end{array}$ & 597.000 & 2985.000 & 0.105 \\
\hline 20 & Piqueteros & 552.000 & 2760.000 & 0.097 \\
\hline 21 & Org. Internac. & 541.000 & 2705.000 & 0.095 \\
\hline 9 & de la Soo. Civil & 496.000 & 2480.000 & 0.087 \\
\hline 17 & Profesionales & 476.000 & 2380.000 & 0.084 \\
\hline 5 & Gob. Nacional & 458.000 & 2290.000 & 0.080 \\
\hline 8 & Sindicatos & 366.000 & 1830.000 & 0.064 \\
\hline $1 \mathrm{Soc}$. & Civil no Organizada & 292.000 & 1460.000 & 0.051 \\
\hline 6 & Gob. Municipal & 259.000 & 1295.000 & 0.046 \\
\hline 3 & Desempleados & 216.000 & 1080.000 & 0.038 \\
\hline 4 & pcia de Cba. & 197.000 & 985.000 & 0.035 \\
\hline 12 & Dacentes & 146.000 & 730.000 & 0.026 \\
\hline 14 & Voluntarios & 124.000 & 620.000 & 0.022 \\
\hline 16 & Niños, Adolescentes & 88.000 & 440.000 & 0.015 \\
\hline 18 & Opinión Pública & 74.000 & 370.000 & 0.013 \\
\hline 7 & Universidades & 74.000 & 370.000 & 0.013 \\
\hline 19 & Justicia & 74.000 & 370.000 & 0.013 \\
\hline 10 & Sector Privado & 22.000 & 110.000 & 0.004 \\
\hline 13 & Alfonsin & 16.000 & 80.000 & 0.003 \\
\hline 11 & Estudiantes & 14.000 & 70.000 & 0.002 \\
\hline
\end{tabular}

Tabla 5. Grado de Centralidad de los actores.

Para continuar con el análisis se hace necesario indagar sobre la centralidad de cada actor dentro de las redes de la pobreza, por lo que se analizará el grado que recibe cada actor mencionado. Se analiza la segunda columna del cuadro porque los valores se encuentran normalizados, operación que permite hacer comparables a los mismos.

El grado de centralidad manifestado permite rescatar por ejemplo a tres actores que presenta los niveles más altos, como lo son los sectores carenciados, la iglesia y los grupos piqueteros. También se puede localizar la centralidad del gobierno nacional, seguido del municipal, y por último del provincial, dato llamativo éste dado que el análisis se efectúa sobre su territorio. Un actor que continua reafirmando la tendencia es el de los estudiantes, donde en otras época de reivindicaciones ocupaba los primeros lugares, y décadas después se localiza en ultimo lugar, sumamente alejado de los sectores populares que lo habían identificado en la época de los 70'. 


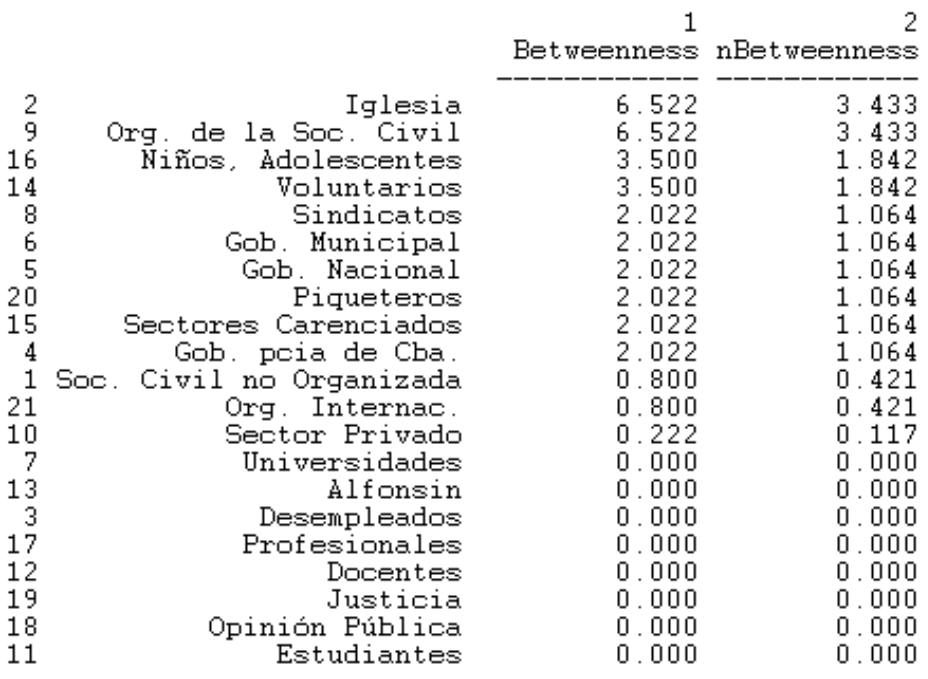

Tabla 6. Grado de intermediación o betweeness.

Este tipo de análisis nos permite observar aquellos actores o nodos que tienen la capacidad de conectar unos actores con otros según los significantes a los cuales menciones, incluso la posibilidad de vincular actores que no se encuentran relacionados directamente por las temáticas a las que se asocian.

En la tabla se puede observar cuatro grupos de actores, el primero y más importante corresponde a la Iglesia y a las Organizaciones de la Sociedad Civil, seguidos por Niños, Adolescentes y Voluntarios. Luego un grupo con menos nivel que es el que corresponde a $n$ Betweenness 1.064, y por último la Sociedad Civil no Organizada, las Organizaciones Internacionales y el Sector Privado. Se presenta también un considerable número de actores (8) que no poseen capacidad de intermediación. Posiblemente responda a que solo remiten a un solo lexema significante, lo que no le permitiría vincular a otros actores, sino solo ser vinculado.

Una técnica importante que permite analizar los posibles grupos de actores que se presenta en este esquema semántico es la de identificar los cliques que se puedan haber producido en la red debido inexistencia de diferencias entre ellos y que la importancia entre ellos es la misma. Cabe la posibilidad de que uno o más actores se encuentren en diferentes cliques. Un primer análisis contempla un tamaño mínimo de 3, y se obtiene lo siguiente: 


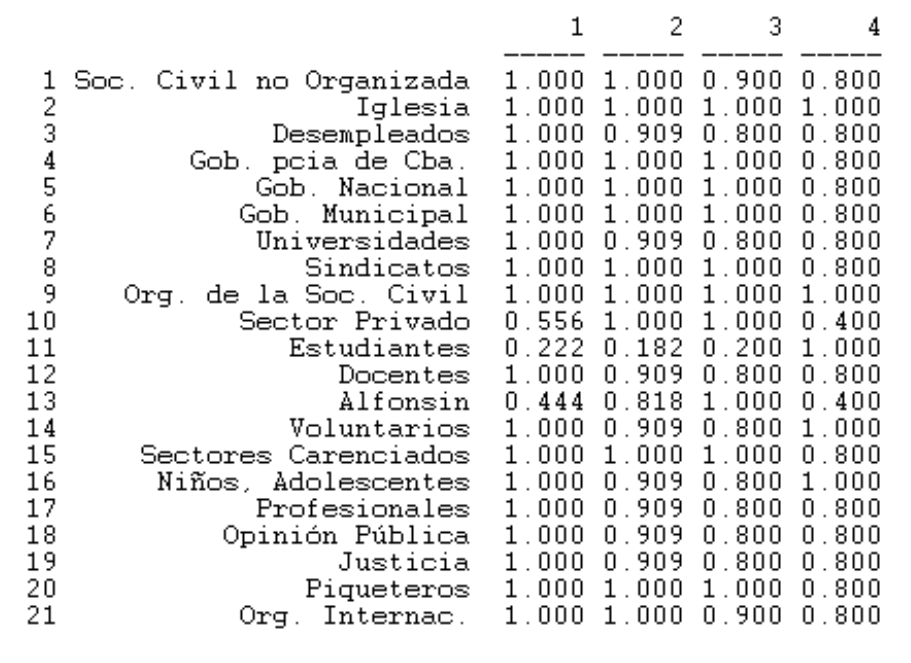

Tabla 7. Cliques de tamaño 3.

La tabla nos muestra cuatro grupos o cliques, donde todos y cada uno de los miembros de un clique son adyacentes entre ellos, es decir que se encuentran conectados. También se presentan actores que se encuentran en más de un clique (resaltados).

1. Soc. Civil no Organizada I glesia Desempleados Gob. pcia de Cba. Gob. Nacional Gob. Municipal Universidades Sindicatos Org. de la Soc. Civil Docentes Voluntarios Sectores Carenciados Niños, Adolescentes Profesionales Opinión Pública J usticia Piqueteros Org. I nternac.

2. Soc. Civil no Organizada Iglesia Gob. pcia de Cba. Gob. Nacional Gob. Municipal Sindicatos Org. de la Soc. Civil Sector Privado Sectores Carenciados Piqueteros Org. I nternac.

3. I glesia Gob. pcia de Cba. Gob. Nacional Gob. Municipal Sindicatos Org. de la Soc. Civil Sector Privado Alfonsin Sectores Carenciados Piqueteros.

4. Iglesia Org. de la Soc. Civil Estudiantes Voluntarios Niños, Adolescentes.

Estos cuatro grupos nos permite observar la poca claridad que presenta trabajar con esta medida incluso si se aumenta su tamaño (se utilizó la prueba hasta 7 y los resultados seguían la misma dirección).

Un análisis de K-Core (no se adjunta gráfico) nos permite observar la conformación de 5 grupos, pero los resultados son los mismos, los actores continúan repitiéndose en más de dos grupos. 
Otro procedimiento que indagar respecto a la estructura semántica de la red es la "modelización en bloques" o como también se le denomina "equivalencia estructural", esto es, siguiendo a Doreian $(1998 ; 11)$ "una estructura simplificada que da forma a la red más grande", los actores se van a encontrar agrupados de acuerdo a la conexión que presenten con el resto de la red, que se supone equivalente. Esta partición de la red permitió dividir la matriz en cuatro bloques y agruparlo de acuerdo a su vinculación en la red. Éstos son:

1. I glesia Sectores Carenciados Piqueteros Org. Internac.

2. Estudiantes Alfonsin.

3. Soc. Civil no Organizada Desempleados Gob. pcia de Cba. Gob. Nacional Gob. Municipal Sindicatos Org. de la Soc. Civil Docentes Voluntarios Profesionales.

4. Universidades Sector Privado Niños, Adolescentes Opinión Pública Justicia.

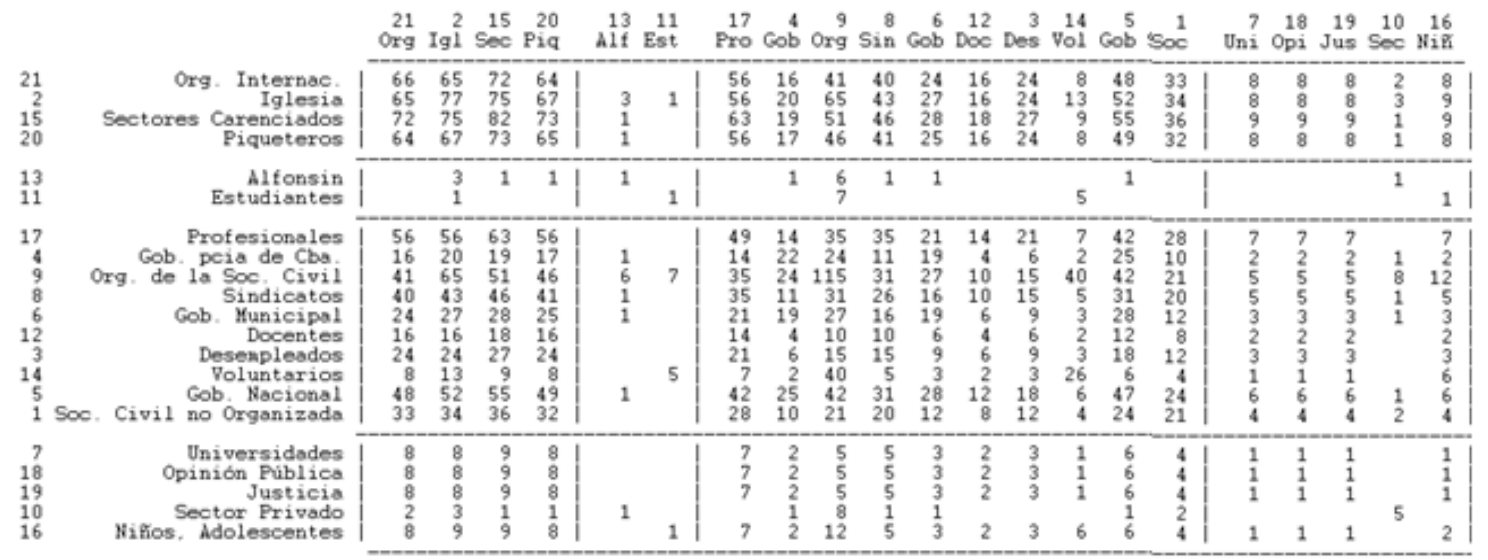

Density Table

\begin{tabular}{rlll}
1 & \multicolumn{1}{c}{2} & \multicolumn{1}{c}{3} & \multicolumn{1}{c}{4} \\
---- & ---- & ---- & ---- \\
69.33 & 0.75 & 33.05 & 7.00 \\
0.75 & 0.00 & 1.10 & 0.20 \\
33.05 & 1.10 & 16.76 & 3.56 \\
7.00 & 0.20 & 3.56 & 0.60
\end{tabular}

Tabla 8. Grupos resultantes de la equivalencia estructural.

Esta tabla nos permite reafirmar la presencia de un grupo de actores centrales, y son los que hasta el momento se han destacado en todos los análisis, el primer grupo analizado es el que mayor densidad posee entre si, lo que nos habla de su cohesividad. A su vez se encuentra relacionado con el tercer grupo, donde se encuentra gran parte de los actores con capacidad de intermediación, pero cuyo nivel de cohesión en relación al primer grupo es bajo.

Por último, se esquematiza lo señalado mediante un gráfico que permite visualizar la red. 


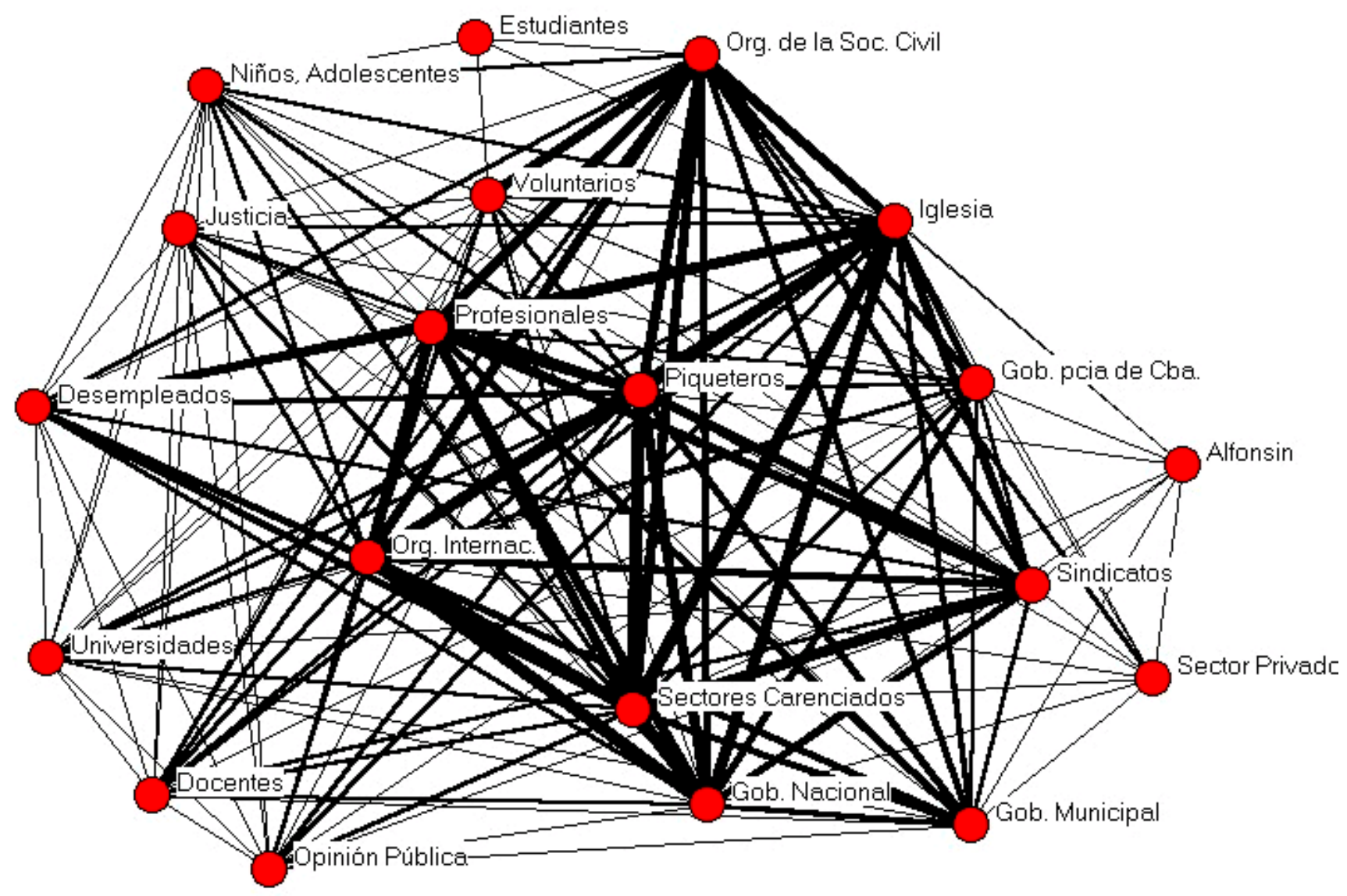

I lustración 1. Grafo de actores por actores.

Lo que continúa en este momento es el análisis de los significantes a los que refieren estos actores.

\begin{tabular}{|c|c|c|c|c|c|c|c|c|c|}
\hline & $\underset{\text { Dem }}{1}$ & $\mathrm{Cr}^{2}$ & $\begin{array}{r}3 \\
\text { Den }\end{array}$ & Den & Of & & & $\begin{array}{r}7 \\
\operatorname{In} i\end{array}$ & Re: \\
\hline Denandas de Rec, bs & 53 & 87 & 4 & 4 & 22 & & & 3 & \\
\hline 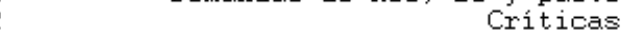 & 87 & 45 & 18 & 14 & 45 & & & 16 & 17 \\
\hline al Esta & 4 & 18 & 6 & 1 & 4 & & 1 & 1 & 2 \\
\hline Denunoias a Movimientos $\mathrm{P}$ & 4 & 1 & 1 & 2 & & & & 1 & \\
\hline Oferta de Politicas Pública & 22 & 4 & 4 & 3 & 38 & & & & \\
\hline Iniciativas y Fal. de la Sac. Civil & 45 & 4 & 1 & 1 & 14 & & & 1 & \\
\hline Inic y Fol. de & 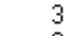 & 10 & & 1 & & & 1 & 5 & \\
\hline Reconocimientos y apoyos a Foliticas & 8 & 17 & 2 & 0 & & & & 1 & \\
\hline
\end{tabular}

Tabla 9. Matriz de temas por temas.

En esta tabla se pueden observar las vinculaciones entre los diferentes temas debido a que coinciden a ser mencionados por los mismos actores. Las vinculaciones que se pueden destacar son: Demandas con Críticas e Iniciativas surgidas en la Sociedad Civil. Críticas con Demandas, Iniciativas de la Sociedad Civil y Ofertas. Ofertas con Críticas y Demandas. Y por último Iniciativas de la Sociedad Civil con Demandas y Críticas. 


\begin{tabular}{|c|c|c|c|}
\hline & Degree & NrmDegree & Share \\
\hline 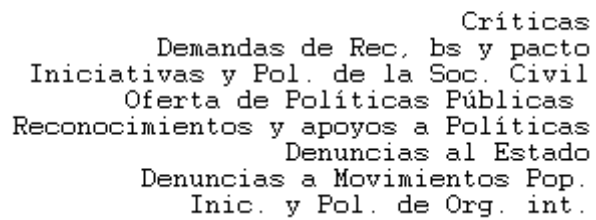 & $\begin{array}{r}246 \cdot 000 \\
173 \cdot 000 \\
118.000 \\
90.000 \\
37.000 \\
31.000 \\
24.000 \\
23.000\end{array}$ & $\begin{array}{r}3514 \cdot 286 \\
2471 \cdot 428 \\
1685 \cdot 714 \\
1285.714 \\
528 \cdot 571 \\
442 \cdot 857 \\
342 \cdot 857 \\
328.571\end{array}$ & $\begin{array}{l}0.332 \\
0.233 \\
0.159 \\
0.121 \\
0.050 \\
0.042 \\
0.032 \\
0.031\end{array}$ \\
\hline
\end{tabular}

Tabla 10. Centralidad de los temas.

Los principales nodos semánticos a los cuales remiten los actores son: Críticas, Demandas, Iniciativas de la Sociedad Civil, y Ofertas de Políticas Públicas.

El hecho de que las demandas se encuentren por encima de la oferta de políticas, puede ser $\mathrm{u}$ indicador de la insuficiencia de estas ante la carencia social que presenta el periodo, más aun si contextualizamos este dato en la época: seis meses después de 2001 los índices de pobreza e indigencia encontrarían en sus filas a más del $50 \%$ de la población. Por otro lado el dato que da "críticas" es otro dato que al contextualizarlo no informa sobre lo que estaba anticipando: una creciente movilización popular que tendría eco en la crisis de 2001.

\begin{tabular}{|c|c|c|}
\hline & Betweenness & nBet weenness \\
\hline Demandas de Rec be y pacto & 0.333 & 1.587 \\
\hline Criticas & 0.333 & 1.587 \\
\hline ias al Estado & & 1.587 \\
\hline Iniciativas y Fol. de la Soo Civil & & 1. 587 \\
\hline Oferta de Foliticas Fúblicas & 0.167 & 0.794 \\
\hline Denuncias a Movimientas Pop. & & 0.794 \\
\hline Inic y Fol de Org int & 0.167 & 0.794 \\
\hline mientos y apoyos a & 0.167 & 0.794 \\
\hline
\end{tabular}

Tabla 11. Capacidad de Intermediación Semántica.

Esta tabla nos muestra dos grupos de temas con capacidad de intermediación entre ellos. Ninguno de los lexemas que desvinculado por lo que se observan en los datos, todos pueden intermediar entre determinados temas o vincular a aquellos que no se encuentren relacionados por medio de algún actor.

\section{Cliques}

La presente técnica nos permite observar la conformación de cuatro grupos, y debido a que el número de nodos involucrados es mucho menos que el anterior, podemos trabajar y analizar las configuraciones estructurales aquí presentes: 


\section{Diferta de Politicas Pública5}

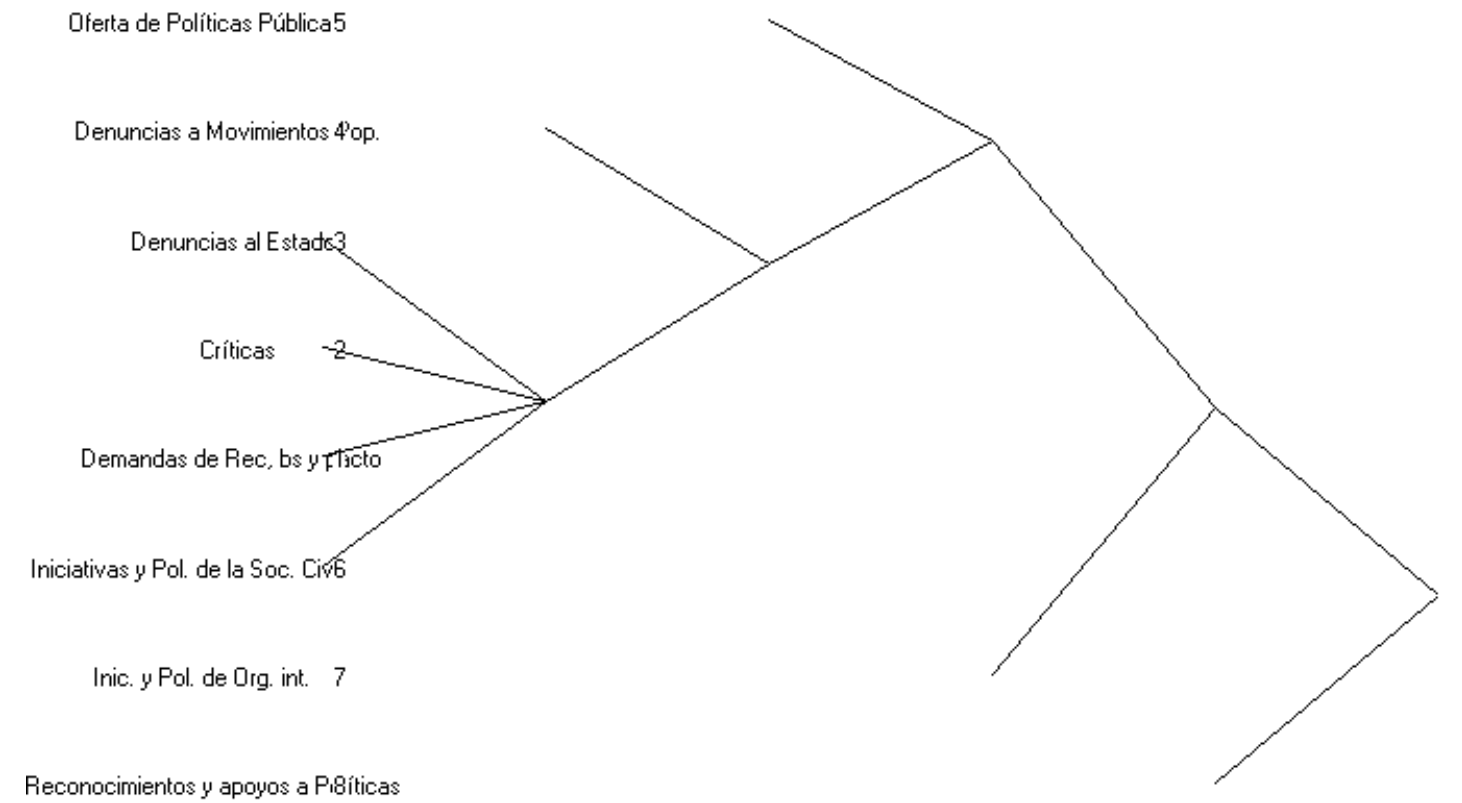

Reconocimientos y apoyos a Pigiticas

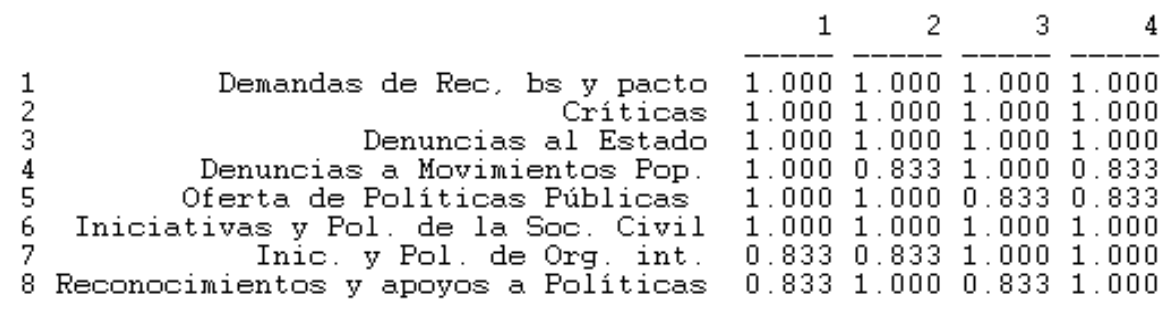

Leyenda:

1. Demandas de Rec, bs y pacto, Críticas, Denuncias al Estado, Denuncias a Movimientos Pop., Oferta de Políticas Públicas, Iniciativas y Pol. de la Soc. Civil.

2. Demandas de Rec, bs y pacto, Críticas, Denuncias al Estado, Oferta de Políticas Públicas, Iniciativas y Pol. de la Soc. Civil, Reconocimientos y apoyos a Políticas.

3. Demandas de Rec, bs y pacto, Críticas, Denuncias al Estado, Denuncias a Movimientos Pop., Iniciativas y Pol. de la Soc. Civil, Inic. y Pol. de Org. int.

4. Demandas de Rec, bs y pacto, Críticas, Denuncias al Estado, Iniciativas y Pol. de la Soc. Civil, Inic. y Pol. de Org. int., Reconocimientos y apoyos a Políticas.

I lustración 2. Asociación estructural de temas.

Se puede observar que el primer clique se vincula con un el quinto tema en el corte 2.000 "Denuncias a movimiento" y recién en el corte 1.833 se evidencia la presencia de la participación estatal, lo que puede permitir afirmar que no solo su capacidad de respuesta no respondía a la estructura de necesidades de la población sino que distaba mucho de ella. 


\section{Modelización en Bloques}

Esta técnica permitió identificar cuatro bloques que luego se incorporarán a la red semántica global:

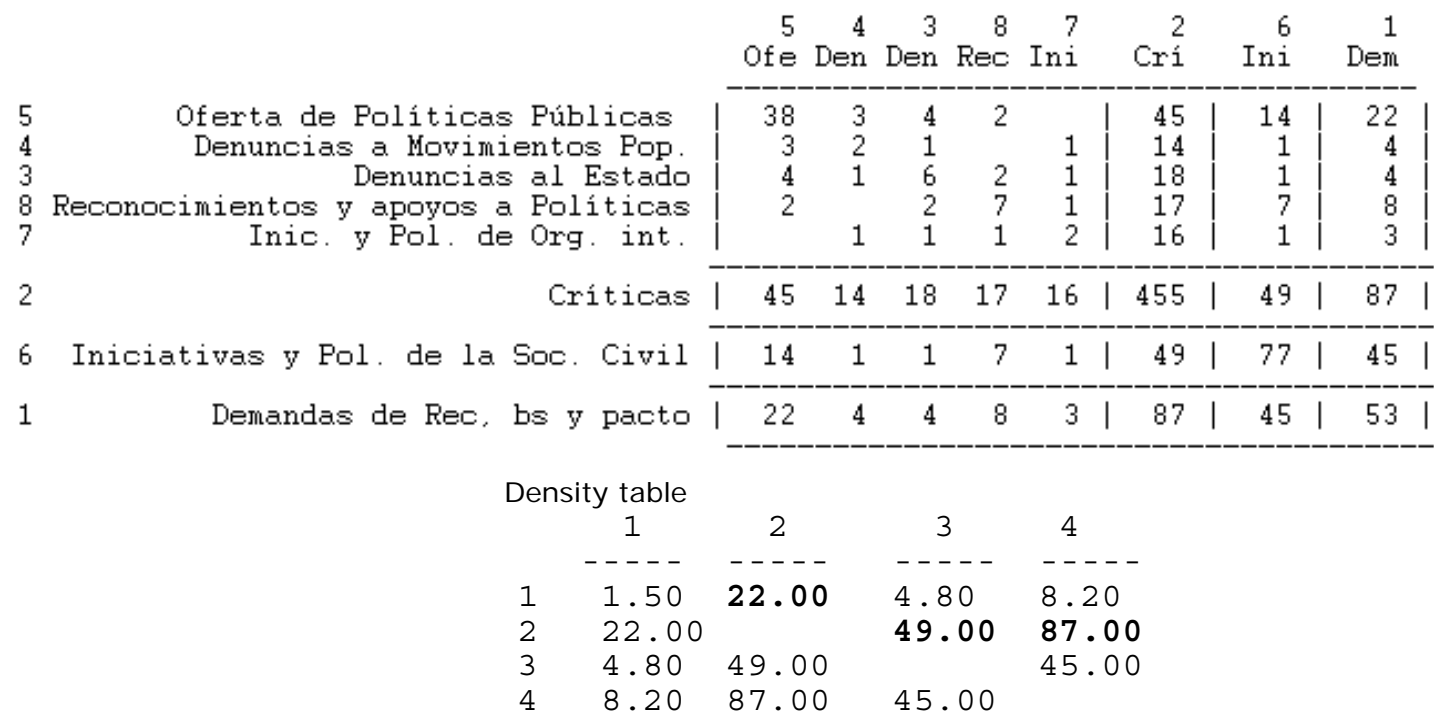

Leyenda:

1. Denuncias al Estado, Denuncias a Movimientos Pop., Oferta de Políticas Públicas, Inic. y Pol. de Org. int., Reconocimientos y apoyos a Políticas.

2. Críticas.

3. Iniciativas y Pol. de la Soc. Civil

4. Demandas de Rec, bs y pacto.

Tabla 12. Bloques de equivalencia estructural.

Los datos no permiten hablar de la existencia de cohesividad dentro de los grupos, si de densidad en cuanto a la relación entre el bloque 1 con el 2, del 2 con el 3 y el 4. Estos datos estarían marcando los consensos en términos discursivos.

Igual que en análisis de actores, a continuación se expone la red de temas. 


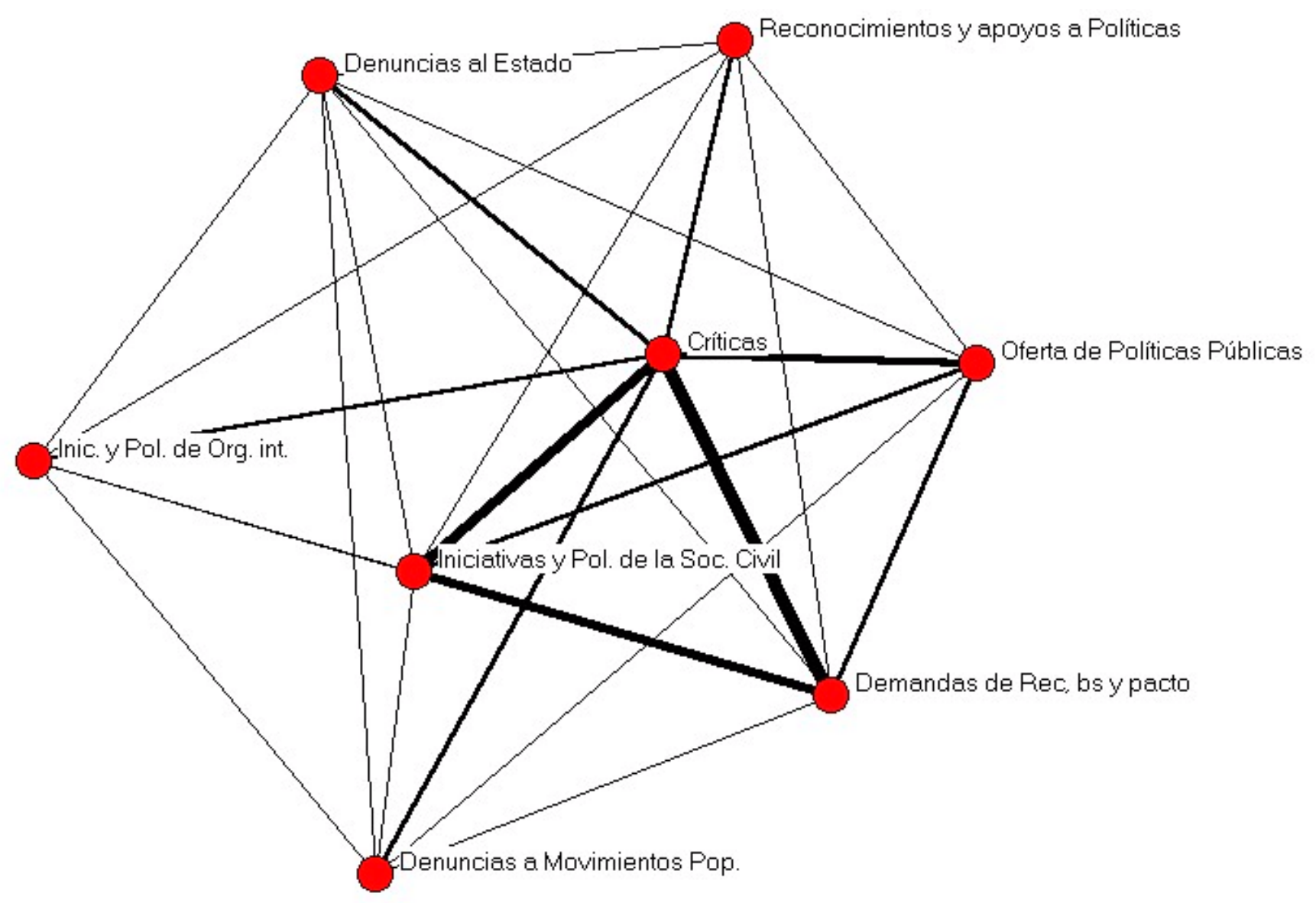

I lustración 3. Grafo de la red de temas.

Después de un análisis por actores y luego por temas (eventos) se intenta vincular a ambos en la red global que estructura la realidad de la pobreza en el año 2001. Este gráfico intenta dar cuenta de la naturaleza reticular que tiene la realidad social y por la cual se estructura (Foucault, 1994). Simultáneamente se intenta demostrar como las diferentes partes de los discursos, en este caso las noticias de la prensa, estructuran de determinada manera la temática que se trabaja. Por último se analiza la red según los principales nodos temáticos y sobre los principales actores (véanse apartados sobre centralidad). Recuérdese que los principales nodos semánticos a los cuales remiten los actores son: Críticas, Demandas, Iniciativas de la Sociedad Civil, y Ofertas de Políticas Públicas. Los principales actores a su vez son los Grupos Piqueteros, Sectores Carenciados, la Iglesia. 


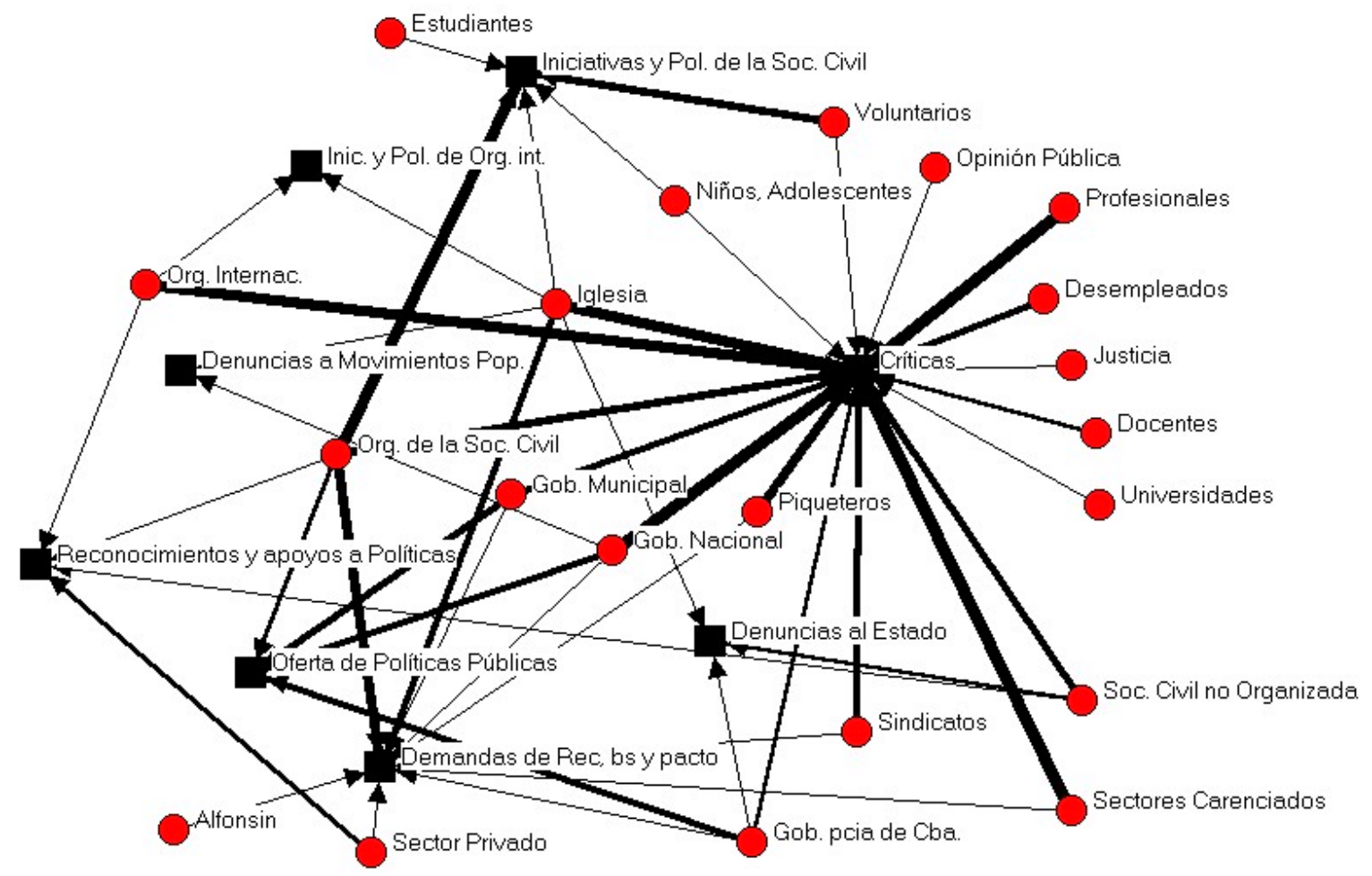

I lustración 4. Red semántica de la pobreza.

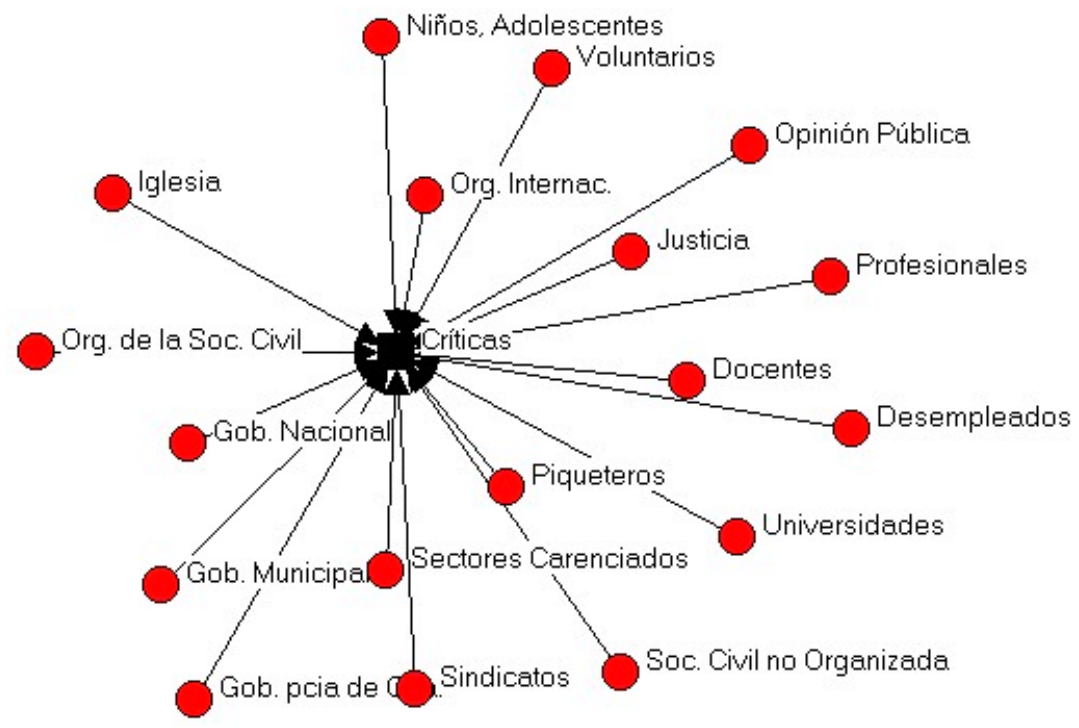

I lustración 5. Red de críticas.

Es evidente la capacidad de movilización que pueden llegar a tener los tres actores centrales Iglesia, Piqueteros y Sectores Carenciados. La activación de cualquiera de los significantes a los que estos se dirijan comienza a articular el resto de la red. Si bien existe un grupo de actores que se encuentra vinculado por un solo significante, la fortaleza del vínculo que tienen los demás actores con el mismo 
(Críticas) permite la activación instantánea y a su vez su capacidad de intermediación.

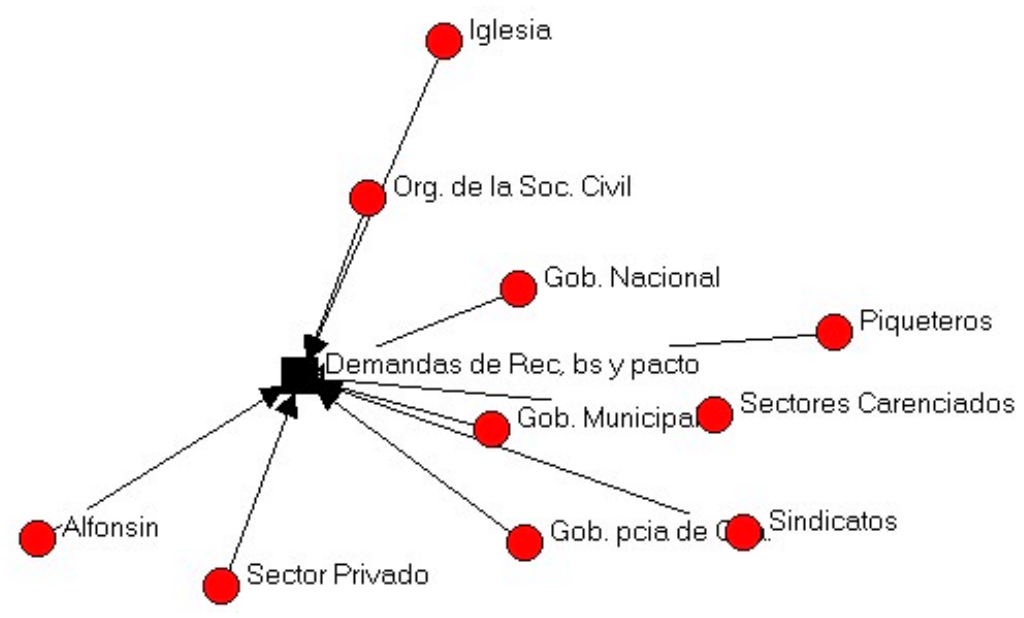

I lustración 6. Demanda de Recursos Bienes y Pacto.

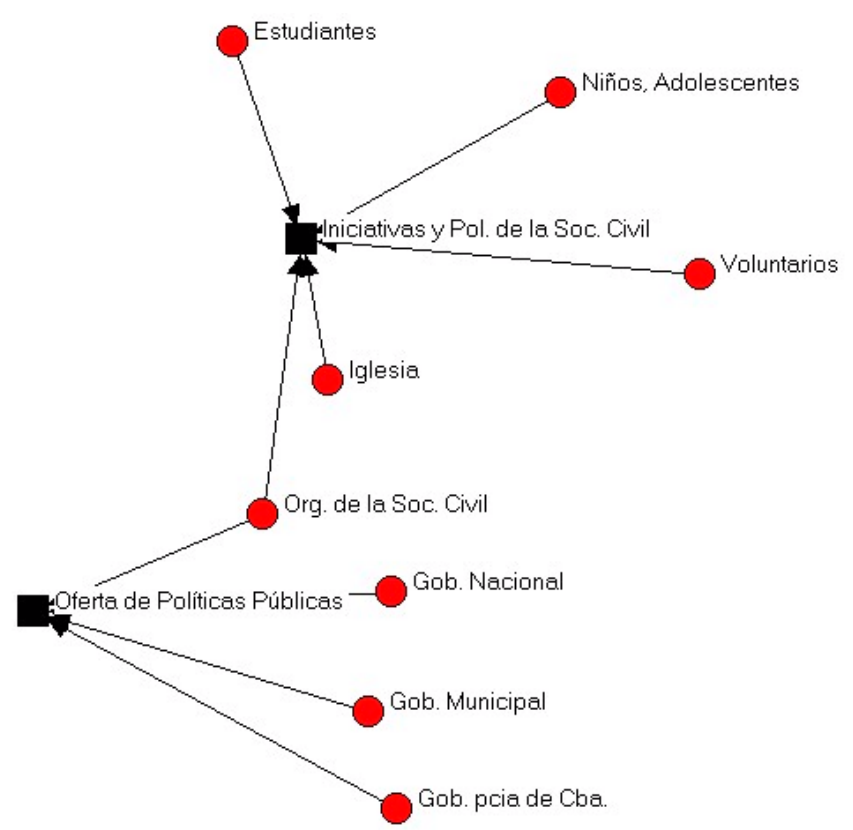

I lustración 7. Iniciativas y Pol. Surgidas en la Soc. Civil - Oferta de Políticas Públicas.

En esta subred se observa claramente la capacidad que tienen las Organizaciones de la Sociedad Civil para intermediar entre el gobierno (cualquiera su nivel) y parte de la sociedad en el Diseño y Gestión de Políticas Públicas. Ni siquiera la I glesia, actor de mayor centralidad, puede mediar en este tipo de temáticas, propias del Estado. 


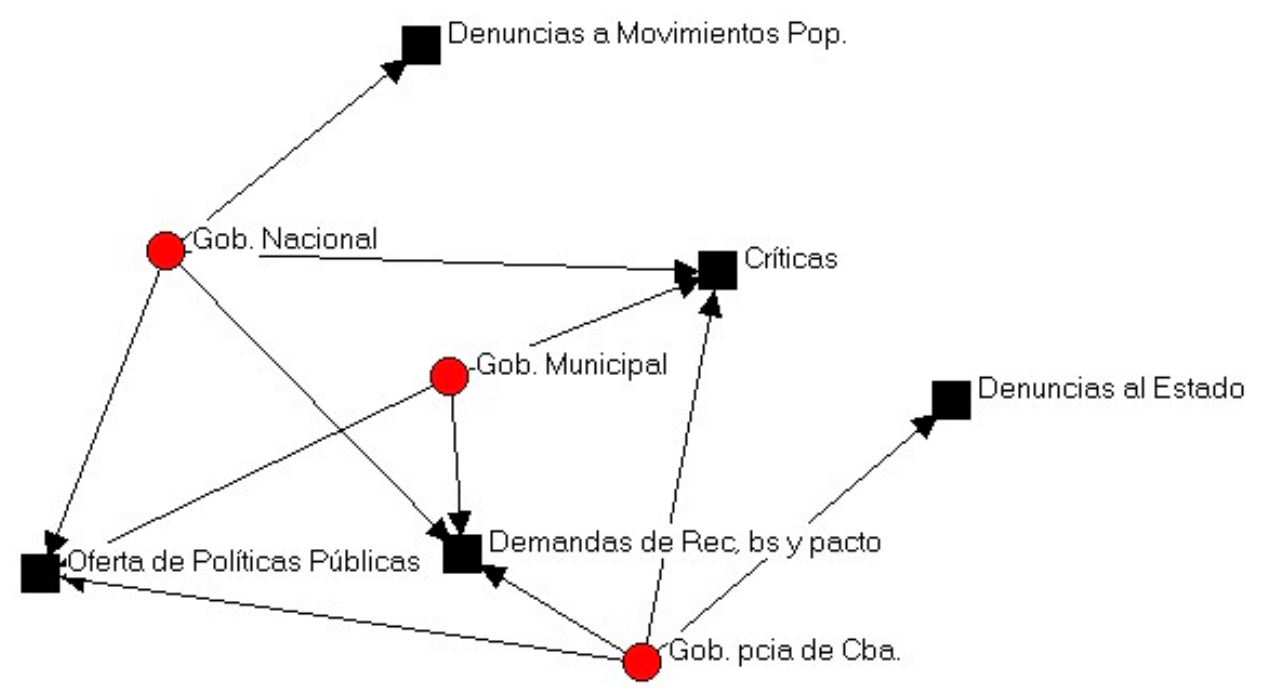

I lustración 8. Gobierno Provincial, Nacional y Municipal.

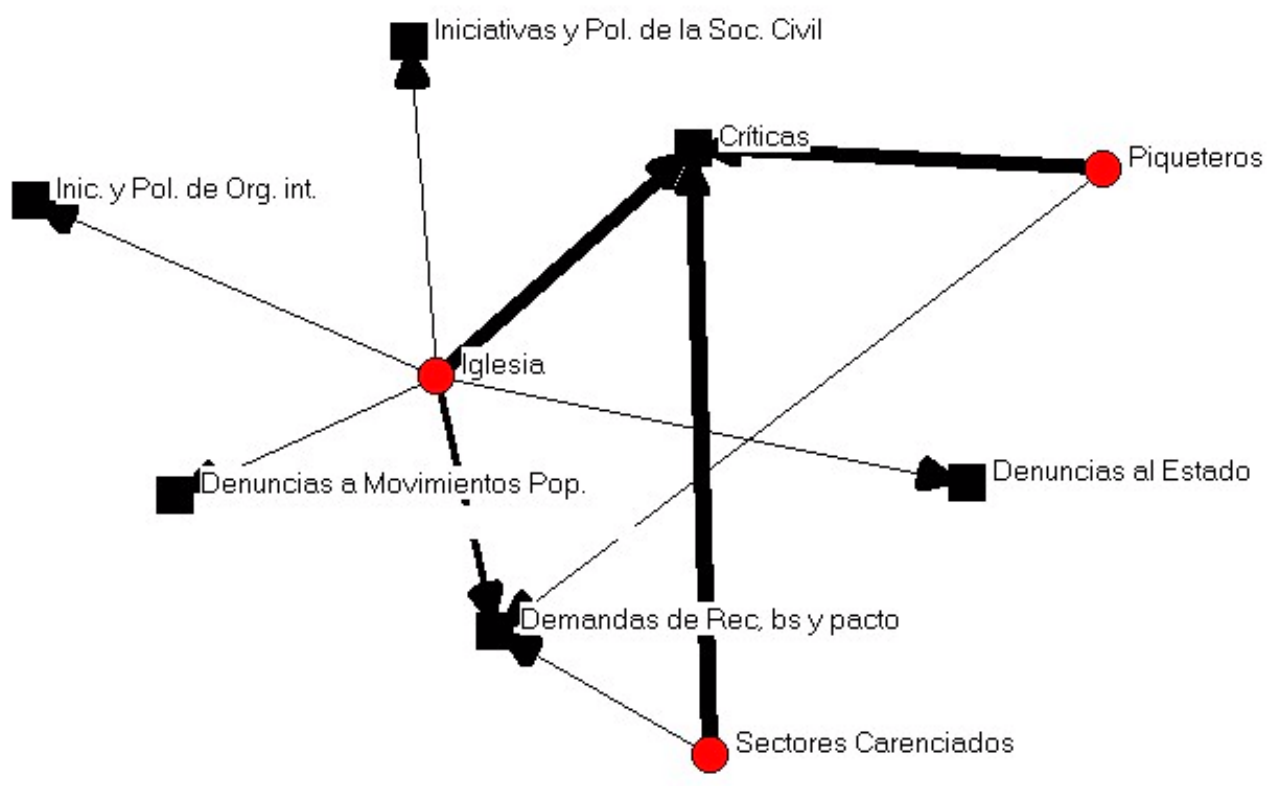

I lustración 9. Grupos Piqueteros, I glesia y Sectores Carenciados.

En este cuadro se puede observar que tanto piqueteros como sectores carenciados se estarían presentando como estructuralmente equivalentes si la red fuera de este tamaño, debido a que las conexiones entre ellos son de las mismas características, ambos Critican o Demandan. En tanto la Iglesia diversifica sus preferencias en una gama más amplia de temáticas. 


\section{Conclusiones}

La transformación de los enunciados en definiciones contextuales permite por la estructura de las mismas, una visualización clara de las similitudes y diferencias entre lo enunciado por los distintos informantes, facilitando el proceso comparativo, tanto manualmente como informáticamente. En este proceso se van elaborando hipótesis de trabajo que conducen a ensayar agrupamientos entre conjuntos de definiciones contextuales, construyendo redes de enunciados (secuenciales, por relaciones de isotopía, y contrastantes). De este modo se determinan ejes conceptuales que identifican a los distintos conjuntos de significado.

Cuando se les interroga sobre sí creen que la democracia es preferible a cualquier otro sistema de gobierno, muestran un apoyo masivo al sistema democrático y sus ideales. Dos terceras partes lo prefieren, y solo un $20 \%$ siguen exhibiendo inclinaciones hacia el autoritarismo. Pero cuando se profundiza expresan que están fuertemente insatisfechos con como la democracia está funcionando en sus países. Solo el 35\% esta satisfecho con su funcionamiento. En la Unión Europea para comparar la cifra es el $47 \%$, en Dinamarca el $84 \%$. Los latinoamericanos han elegido la democracia como forma de vida, y la respaldan consistentemente, pero "democráticamente" están fuertemente disconformes con su desempeño concreto (Latinobarómetro, 1999).

Entre las causas de insatisfacción algunas son políticas, pero tienen un peso decisivo las económico-sociales. La gran mayoría considera que los problemas vinculados con la pobreza han empeorado. Se refieren a carencias en oportunidades de trabajo, acceso a salud, acceso a educación de buena calidad, incertidumbre laboral, bajos sueldos. Agregan a ello temas como el agravamiento de la corrupción, la delincuencia, y el tráfico de drogas. Además, testimonian que sienten que ésta es una región donde existen grandes desigualdades y resienten agudamente esa situación.

La teorización sobre las formas de desigualdad económica o material ha dado lugar a profundadas indagaciones sobre la potencialidad que ofrece el concepto de pobreza, como una forma de opresión, entre otras. Estos abordajes van desde indagaciones en base a mediciones exhaustivas en término de acceso a determinados bienes y servicios, e ingresos, pasando por las condiciones de privación, marginación y exclusión, hasta su localización y dinámica temporal y espacial a través del tiempo. sin desconocer estos valiosos enfoques, optamos por mostrar la potencialidad que reviste el análisis de redes aplicado a la producción 
textual, particularmente a notas periodísticas de difusión masiva que tematizan sobre la pobreza

Como señalábamos, sin desconocer estos enfoques, esta investigación se distancia de ellos a nivel teórico como metodológico, en tanto no predefinimos un concepto a priori sobre la pobreza, para así poder captar toda la diversidad que sobre este se congrega y por otro lado abordar el concepto desde un encuadre metodológico lo suficientemente flexible como para comprender la dinámica que acontece en su construcción. En este sentido, el texto aparece como la referencia discursiva que informa sobre el sentido del concepto, e ilumina otras particularidades o aspectos, indagados menos intensamente. El análisis de redes permitió reconstruir esta multiplicidad de sentidos en numerosas redes semánticas que dan cuenta de la complejidad del mismo.

El período analizado reclamaba también un abordaje novedoso. Hoy uno puede leer, a manera retrospectiva estos datos y observar la potencia que posee el análisis como estrategia metodológica para el abordaje de los conflictos sociales. Recordemos que a finales del año 2001 Argentina entró en un creciente estado de conmoción social que se evidenció en el estallido del "19 y 20" de diciembre. Posteriormente a este acontecimiento, los niveles de pobreza estallaron expresando la crisis de un modelo de acumulación gestado desde los años de la última dictadura, y la crisis institucional demandó la renovación de numerosos espacios de dirección, electivos y no electivos, para garantizar la mínima gobernabilidad. Los actores que observáramos en las redes acaparando el centro de la conflictividad social, son los que en los años siguientes a esta crisis dominarán el escenario político y demandarán al Estado y el sector privado una fuerte redefinición. Actualmente las instituciones se han reconfigurado, pero permanecen en ellas lógicas rechazadas fuertemente por diferentes sectores sociales.

\section{Bibliografía}

Auyero, J. (2002). La protesta. Retratos de la beligerancia popular en la Argentina democrática. Serie Extramuros. Universidad de Buenos Aires. Buenos Aires.

Foucault, M. (1998). Microfísica del poder. Madrid: La Piqueta.

Lozano, J., Peña Marín, C., y Abril G. (1997). Análisis de discurso. Hacia una semiótica de la interacción textual. Barcelona: Ediciones Cátedra. 
Lozares Colina, et.at. (2002.) "Relaciones, redes y discurso: revisión y propuestas en torno al análisis reticular de datos textuales", REDES : Revista Hispana para el Análisis de Redes Sociales Vol. 1 \#2. http://revista-redes.rediris.es

Taddei, E. (2003). “Las protestas sociales en el espacio urbano: trabajadores asalariados y convergencias sectoriales". En Revista OSAL. Buenos Aires.

Tarrow, S. (1997). El poder en movimiento. Los movimientos sociales, la acción colectiva y la política. Madrid: Alianza editorial.

Zibechi, R. (2003). Genealogía de la revuelta. Argentina: la sociedad en movimiento. Buenos Aires: Letra Libre. 